Results: Blockade of SHH by GDC-0449 significantly alleviated the symptoms and decreased the synovial hyperplasia, inflammatory infiltration, cartilage and bone damage in ankles of CIA. The bone erosions in the area of the metatarsophalangeal and ankle joints and production of TNFa, IL-6 were decreased by $\mathrm{SHH}$ inhibition. In addition, the administration of GDC-0449 significantly decreased the number of TRAP positive cells and the expression of NFATc1. On the contrary, $\mathrm{SHH}$ overexpression led to increased severity of arthritis and pathological changes. We also observed the accelerated bone injury accompanied with increased number and activity of osteoclasts and increased production of serum IL-6 in mice with upregulation of $\mathrm{SHH}$ expression. Of note, the administration of p38 MAPK inhibitor reversed the effects of SHH overexpression, with a reduction of joint swelling and histological scores. Inhibition of p38 MAPK prevented the bone erosion and decreased the number of TRAP positive cells and the expression of NFATc1, which were promoted by $\mathrm{SHH}$ overexpression.

Conclusion: The study indicates that $\mathrm{SHH}$ promotes the synovial hyperplasia and bone erosion of CIA in a p38 MAPK-dependent manner. SHH-p38 MAPK signaling could be a potential target for the treatment of RA.

Acknowledgments: This work was supported by grants from the National Natural Science Foundation of China (81571584, 81701609).

Disclosure of Interests: None declared

DOI: 10.1136/annrheumdis-2020-eular.3632

\begin{tabular}{|l|l}
\hline THU0077 & CADHERINS GUIDE THE DIRECTIONAL \\
MIGRATION OF THE SYNOVIOCYTES IN \\
RHEUMATOID ARTHRITIS
\end{tabular}

J. H. Kang ${ }^{1}$, S. Ahamed ${ }^{1}$, K. H. Sa ${ }^{1}$, S. W. Han ${ }^{1}$, Y. M. Kang ${ }^{1} .{ }^{1}$ Kyungpook National University School of Medicine, Daegu, Korea, Rep. of (South Korea)

Background: Aggressiveness of synoviocytes and collective migration of organized synovial tissues play a key role in the pathogenesis of pannus invasion into adjacent joint structure. Interactions among synovial cells for grouped movement, however, have not been properly elucidated.

Objectives: We hypothesized that cadherins which have functions on the synovial invasion in RA, may play a critical role in collective migration of rheumatoid synoviocytes.

Methods: Cadherins expression patterns on the synoviocytes isolated from patients with RA were evaluated using RT-PCR, flow cytometry, and western blot analysis. Mesenchymal and epithelial phenotypes were examined in cadherin overexpressing cell line by flow cytometry. L-cells with overexpression of $\mathrm{CDH} 2\left(\mathrm{CDH} 2^{\mathrm{hi}}\right), \mathrm{CDH} 11$ (CDH11 ${ }^{\mathrm{hi}}$, and combination of $\mathrm{CDH} 2 / \mathrm{CDH} 11\left(\mathrm{CDH} 2^{\mathrm{hi}}\right.$ ) $\left.\mathrm{CDH} 11^{\text {hi }}\right)$ were prepared. Migration of cells was observed by taking time-lapse images with laser confocal microscope. In vitro collective migration and directional movement in response to inflammatory mediators and different matrix rigidity were evaluated. In vivo homing of $\mathrm{CDH} 2^{\text {hi }} / \mathrm{CDH} 11^{\text {hi }}$-L-cells into joint tissues was performed in collagen induced arthritis (CIA) mouse. In vivo and ex vivo migration pattern of $\mathrm{CDH} 11^{\mathrm{hi}}$-L-cells were investigated in nude mice using optical imaging system.

Results: In rheumatoid synovial tissues, $\mathrm{CDH} 2$ and $\mathrm{CDH} 11$ were highly expressed compared to synovial tissues from osteoarthritis. $\mathrm{CDH} 2$ and $\mathrm{CDH} 11$ were also highly expressed on synovial fibroblasts isolated from RA. Phenotype analysis of mesenchymal and epithelial cells in $\mathrm{CDH} 11^{\text {hi }}$-L-cells and $\mathrm{CDH} 2{ }^{\text {hi }} / \mathrm{CDH} 11^{\text {hi }}$-L-cells showed increased expression of $a 5 \beta 1, \mathrm{CD} 44 \mathrm{~s}$, vimentin, and a-SMA compared with MOCK-L-cells. We then analyzed the pattern of migration of MOCK, CDH2 $2^{\text {hi }}, \mathrm{CDH} 11^{\text {hi }}$, and $\mathrm{CDH} 2^{\text {hi }} / \mathrm{CDH} 11^{\text {hi }}$-L-cells using time lapse images. During migration over a hard ECM, CDH2 ${ }^{\text {hi }}$ and $\mathrm{CDH} 11^{\mathrm{hi}}-\mathrm{L}$ cells represented higher aspect ratio compare to a soft ECM. Aspect ratio relatively found lower in $\mathrm{CDH} 2^{\text {hi }} / \mathrm{CDH} 11^{\text {hi }}$-L-cell lines than MOCK cells. CDH2 ${ }^{\text {hi }}$ $\mathrm{CDH} 11^{\text {hi }}$-L-cells showed significantly higher migration velocity and Euclidean distance with narrower angle of migratory directions in a cytokine mediated migration. Compared to the MOCK cells, persistence ratio and aspect ratio of migration were also higher in $\mathrm{CDH} 2^{\mathrm{hi}}, \mathrm{CDH} 11^{\mathrm{hi}}$, and $\mathrm{CDH} 2^{\mathrm{hi}} / \mathrm{CDH} 11^{\mathrm{hi}}$-L-cells. $\mathrm{CDH} 2^{\text {hi }} / \mathrm{CDH} 11^{\text {hi }}$-L-cells collectively migrated with the formation of leader and follower cells. In a chemokine mediated hard stiffness of ECM, durotaxis was observed in $\mathrm{CDH} 11^{\mathrm{hi}}$-L-cells. After 24 hours of intraarticular knee injection in $\mathrm{CIA}$ mouse, higher number of $\mathrm{CDH} 2^{\text {hi }} / \mathrm{CDH} 11^{\text {hi }}$-L-cells invaded into the cartilage than MOCK cells. In vivo migration of $\mathrm{CDH} 2^{\text {hi }} / \mathrm{CDH} 11^{\text {hi }}$-L-cells was also found towards the chemokine and cartilage mixed matrigel plug in the subcutaneous space of mice.

Conclusion: The expression of $\mathrm{CDH} 2$ and $\mathrm{CDH} 11$ promotes directional migration of synoviocytes, indicating the potential role of these cadherins on the pannus tissues in the invasion into adjacent joint structure in RA.

References:

[1] Noss EH, Chang SK, Watts GFM, Brenner MB. Cadherin-11 engagement modulates matrix metalloproteinase production by rheumatoid arthritis synovial fibroblasts. Arthritis Rheum. 2011 Dec; 63(12): 3768-3778
Disclosure of Interests: None declared

DOI: 10.1136/annrheumdis-2020-eular.6426

\begin{tabular}{ll}
\hline THU0078 & EXPRESSION PROFILE ANALYSIS OF LONG \\
& NONCODING RNAS INDUCED BY IL-1SS IN \\
& RHEUMATOID ARTHRITIS FIBROBLAST-LIKE \\
& SYNOVIOCYTES
\end{tabular}

J. M. Kim ${ }^{1}$, H. J. Kang ${ }^{2}$, S. J. Jung ${ }^{3}$, B. W. Song ${ }^{1}$, H. J. Jeong ${ }^{1}$, C. N. Son ${ }^{1}$, S. H. Kim ${ }^{1}$, K. Hur ${ }^{4} .{ }^{1}$ Keimyung University Dongsan Medical Center, Keimyung University College of Medicine, Division of Rheumatology, Department of Internal Medicine, Daegu, Korea, Rep. of (South Korea); ${ }^{2}$ Graduate School, Keimyung University, Department of Internal Medicine, Daegu, Korea, Rep. of (South Korea); ${ }^{3}$ Keimyung University School of Medicine, Department of Anatomy, Daegu, Korea, Rep. of (South Korea); ${ }^{4}$ School of Medicine Kyungpook National University, Department of Biochemistry and Cell Biology, Cell and Matrix Research Institute, Daegu, Korea, Rep. of (South Korea)

Background: Long noncoding RNAs (IncRNAs) have recently emerged as important biological regulators and the aberrant expression of IncRNAs has been reported in various diseases including cancer, cardiovascular disease, and diabetes mellitus. However, the role of IncRNAs in the pathogenesis of rheumatoid arthritis (RA) remains unknown.

Objectives: Thus, we studied IncRNAs influenced by IL-1, which is one of the key mediators in the pathogenesis of RA, and also investigated whether regulation of NF-kB activation, which is known to be induced by IL-1, could lead to the changes of expression of those IncRNAs.

Methods: Fibroblast-like synoviocytes (FLS) were obtained from the knee joints of the patients with RA. The next-generation sequencing (NGS) data were analyzed to identify differentially expressed IncRNAs between unstimulated RA FLS and IL-1-stimulated RA FLS. The expression levels of the top 5 candidates in NGS data were validated by RT-qPCR using extended number of unstimulated RA FLS and IL-1-stimulated RA FLS. IMD-0560, an inhibitor of IKB kinase (IKK) was used for the regulation of NF-KB activation. Activation and inhibition of NF-KB were confirmed by Western blotting. Changed expressions of the IncRNAs were identified by RT-qPCR.

Results: NGS analysis revealed up-regulated 30 IncRNAs and down-regulated 15 IncRNAs in IL-1-treated RA FLS compared with unstimulated RA FLS. Top 5 IncRNAs were selected among 30 IncRNAs up-regulated by IL- 1 in RA FLS based on fold-change with P-value cutoff. The up-regulated IncRNAs including NR_046035, NR_027783, NR_033422, NR_003133, and NR_049759 were validated by RT-qPCR. IMD- 0560 inhibited phosphorylation of IKBa induced by IL-1 in RA FLS. Overexpression of IncRNAs induced by IL-1 was also inhibited by IMD-0560 in RA FLS.

Conclusion: Our study revealed that IL-1 increased the expression of NR_046035, NR_027783, NR_033422, NR_003133, and NR_049759 in RA FLS. In addition, the expression of these IncRNAs was regulated by inhibition of NF-kB activation. Thus, our data suggest that the IncRNAs might be involved in the pathogenesis of RA through NF-KB signaling pathway.

References:

[1] Long noncoding RNAs and human disease. Trends Cell Biol. 2011 Jun;21(6):354-61.

[2] A long noncoding RNA mediates both activation and repression of immune response genes. Science. 2013 Aug 16;341(6147):789-92.

[3] Long noncoding RNA expression profile in fibroblast-like synoviocytes from patients with rheumatoid arthritis. Arthritis Res Ther. 2016 Oct 6;18(1):227.

Disclosure of Interests: None declared

DOI: 10.1136/annrheumdis-2020-eular.3072

\section{THU0079 \\ THE MICROBIOME OF NEW-ONSET RHEUMATOID ARTHRITIS (NORA) PATIENTS DRIVES TLR4- DEPENDENT TH17 RESPONSES}

M. Koenders ${ }^{1}$, H. Evans-Marin ${ }^{2}$, J. Aarts ${ }^{1}$, P. Girija ${ }^{2}$, R. Rogier ${ }^{1}$, S. Koralov², J. Manasson ${ }^{2}$, P. Van der Kraan ${ }^{1}$, S. Abdollahi-Roodsaz ${ }^{1,2}$, J. Scher ${ }^{2}$.

${ }^{1}$ Radboud University Medical Center, Experimental Rheumatology, Nijmegen, Netherlands; ${ }^{2}$ New York University School of Medicine, New York, United States of America

Background: Intestinal microbiota plays a prominent role in shaping the $\mathrm{T}$ cell immune response. Increasing evidence suggests that the gut microbiota is perturbed in patients with RA, and a variety of animal models demonstrated involvement of (mouse) microbiota in arthritis development. This underlines the necessity of understanding whether and how indigenous human NORA-associated microbiota may trigger RA. 
Objectives: To comprehensively investigate the intestinal mucosa cytokine production and $\mathrm{DC}, \mathrm{T}$ and $\mathrm{B}$ cell responses to human gut microbiota associated with new-onset RA.

Methods: We utilized in vitro cultures of mucosal-like DCs (differentiated from bone marrow cells) and primary splenic DCs, as well as ex vivo cultures of healthy human intestinal biopsies, cultured in the presence of heat-killed fecal microbiota from either NORA or control donors. Furthermore, we performed studies in humanized mice carrying intestinal NORA microbiota, to study the effect on immune response during homeostasis and upon joint inflammation during collagen-induced arthritis (CIA).

Results: In 24h DC cultures, NORA fecal microbiota more potently induced the expression of co-stimulatory molecules CD40 and CD80, and this enhanced DC maturation was partially mediated through TLR4 as demonstrated using the TLR4 antagonist TAK242. Interestingly, HC and NORA fecal microbiota differentially induced IL-12 and IL-6 production, with significantly enhanced IL-6 and reduced IL-12 secretion by the NORA microbiome. Furthermore, in ex vivo cultures of human ileum biopsies, the production of IL-1 and IL-33, as well as IL-23/Th17 cytokines IL-23, IL-22, and GM-CSF, were significantly increased by NORA-derived microbiome. Interestingly, in the small intestine lamina propria (SILP) of NORA-colonized mice, we observed enhanced Th17 polarization, increased innate GM-CSF expression and higher B cell CD40 and IgA levels during homeostasis. To study whether colonization with $\mathrm{HC}$ and NORA microbiota alters arthritis development, humanized mice and controls (mock, autologous, $\mathrm{HC}$ and NORA microbiota) were used in a CIA experiment. Macroscopic scoring of the arthritis severity at weekly intervals demonstrated that arthritis severity was significantly enhanced in NORA-colonized mice compared to HC-colonization and mock controls.

Conclusion: Our data reveal that NORA microbiota, in addition to the previously described Th17 differentiation, induce higher levels of GM-CSF and B cell $\lg A$ in LP and have increased potential to aggravate arthritis through the activation of TLR4.

References:

[1] Scher et al., eLife 2013; Maeda Y et al., Arthritis \& rheumatology 2016; Zhang

X et al., Nature medicine 2015; Chen J et al., Genome Med 2016

Disclosure of Interests: Marije Koenders: None declared, Heather EvansMarin: None declared, Joyce Aarts: None declared, Parvathy Girija: None declared, Rebecca Rogier: None declared, Sergei Koralov: None declared, Julia Manasson: None declared, Peter van der Kraan: None declared, Shahla Abdollahi-Roodsaz: None declared, Jose Scher Consultant of: Novartis, Janssen, UCB, Sanofi.

DOI: 10.1136/annrheumdis-2020-eular.5269

\section{THU0080 PRECLINICAL CHARACTERIZATION OF TLL018, A NOVEL, HIGHLY POTENT AND SELECTIVE JAK1/TYK2 INHIBITOR FOR TREATING AUTOIMMUNE DISEASES}

X. Liu ${ }^{1}$, F. Tan ${ }^{1}$, C. Liang ${ }^{1} .{ }^{1} T L L$ Pharmaceutical LLC, Iselin, United States of America

Background: Janus kinases (JAKs) are important regulators of intracellular responses triggered by many key proinflammatory cytokines and are clinically validated therapeutic targets for treating various autoimmune diseases. However, current approved JAK inhibitors failed to achieve maximal clinical benefit in part due to their unfavorable selectivity for individual JAKs such as JAK2 and/ or JAK3, leading to dose-limiting toxicities or severe toxicities (e.g., thrombosis, anemia, immune suppression). Selective inhibition of JAK1 and/or TYK2 may minimize or avoid some of the toxicities and potentially offer a better therapeutic window for treating autoimmune diseases. No highly selective JAK1/TYK2 inhibitor has been reported to date.

Objectives: Discovery of a highly selective JAK1/TYK2 inhibitor that maximally avoids JAK2 and JAK3 inhibition. We described preclinical characterization of a novel, highly potent and selective JAK1/TYK2 inhibitor TLL018 and its potential utility in treating autoimmune diseases such as rheumatoid arthritis (RA).

Methods: Using predicting SAR, TLL018 was designed to achieve exquisite selectivity for both JAK1 and TYK2 while sparing JAK2, JAK3 and other human kinases. Its enzyme and cell activities, kinase selectivity, and in vivo efficacy were assessed in a battery of relevant enzyme, cell and whole blood assays, and in vivo arthritis animal models. Additional preclinical DMPK and toxicology studies were conducted to support its clinical development.

Results: TLL018 is a highly potent and selective, orally bioavailable JAK1/TYK2 inhibitor against JAK1 $\left(\mathrm{IC}_{50}=4 \mathrm{nM}\right)$ and TYK2 $\left(\mathrm{IC}_{50}=5 \mathrm{nM}\right)$ as measured in in vitro kinase assays with ATP concentrations at individual $\mathrm{Km}$. Its potency against JAK2 or JAK3 is greater than $1 \mu \mathrm{M}$. Profiling against a panel of over 350 human kinase showed that TLL018 is exclusively selective for JAK1 and TYK2, with $\geq$ 90-fold selectivity against all other kinases tested. TLL018 exhibited potent cellular activity for JAK1-mediated IL-6 signaling $\left(\mathrm{IC}_{50}=0.6 \mu \mathrm{M}\right)$ with greater than 100fold selectivity against JAK2-mediated cytokine (e.g., TPO) signaling in human whole blood-based assays.

Oral administration of TLL018 demonstrated dose-dependent efficacy in commonly studied rat adjuvant-induced arthritis (rAIA) model and mouse collagen-induced arthritis ( $\mathrm{mClA}$ ) model. Significant inhibition of inflammation, bone resorption, splenomegaly and body weight change was observed in adjuvant-induced disease in rats. In addition, significant inhibition of inflammation, cartilage destruction, bone resorption and histological signs was demonstrated in collagen-induced arthritis in mice. Noticeably, TLL018 exhibited significant anti-inflammation activity at doses that only blocked JAK1 and TYK2 and exerted little inhibition of JAK2 and JAK3.

In support of clinical development of TLL018, preclinical ADME and PK studies and IND-enabling toxicology and safety pharmacology studies were completed confirming that TLL018 possesses excellent ADME and PK properties, and exhibits a clean on-target safety profile.

Conclusion: TLL018 is a highly potent and selective JAK1/TYK2 inhibitor that demonstrated excellent efficacy and tolerability in relevant mouse and rat arthritis models. The collective data of its preclinical pharmacology, PK and toxicology showed a favorable pharmaceutical profile, further supporting its development for treating autoimmune diseases including RA. Clinical evaluation of TLL018 is ongoing.

Disclosure of Interests: Xiangdong Liu Shareholder of: I own shares of TLL Pharmaceutical LLC, Employee of: I am employed by TLL Pharmaceutical LLC Fenlai Tan Shareholder of: I own shares of TLL Pharmaceutical LLC, Employee of: I am employed by TLL Pharmaceutical LLC, Chris Liang Shareholder of: I own shares of TLL Pharmaceutical LLC, Employee of: I am employed by TLL Pharmaceutical LLC

DOI: 10.1136/annrheumdis-2020-eular.1547

\section{THU0081 MIR-17-5P REDUCES INFLAMMATION AND BONE EROSIONS IN COLLAGEN INDUCED ARTHRITIS MICE AND DIRECTLY TARGETS THE JAK-STAT PATHWAY IN RHEUMATOID ARTHRITIS FIBROBLAST-LIKE SYNOVIOCYTES.}

A. Najm ${ }^{1,2}$, F. M. Masson ${ }^{1}$, P. Preuss ${ }^{1,2}$, S. Georges ${ }^{1}$, B. Ory ${ }^{1}$, T. Quillard $^{1}$, D. Veale ${ }^{3}$, U. Fearon ${ }^{4}$, B. Le Goff ${ }^{1,2}$, F. Blanchard ${ }^{1}{ }^{1}$ Nantes Faculty of Medicine, PHY-OS Laboratory INSERM UMR 1238, Nantes, France; ${ }^{2}$ Nantes University Hospital, Department of Rheumatology, Nantes, France; ${ }^{3}$ The Centre for Arthritis and Rheumatic Diseases, St. Vincent's University Hospital, Department of Rheumatology, Dublin, Ireland; ${ }^{4}$ Trinity Biomedical Sciences Institute, Trinity College Dublin., Molecular Rheumatology, Dublin, Ireland

Background: micro-RNAs (miR) are strong regulators of gene expression. Their involvement in RA key cytokines pathway regulation entitles them as importan players in RA pathophysiology. The miR 17-92 cluster has been widely studied in cancer as they regulate cell apoptosis.

Objectives: The aims of this study were to screen miR 17-92 cluster's expression in different RA phenotypes (erosive and non erosive), further elucidate the mechanisms and direct targets involved in miR-17-5p anti-inflammatory role and to investigate miR-17-5p therapeutic effect in arthritis.

Methods: A miR array was performed in synovial tissue from naïve erosive and non-erosive RA patients. Intra-articular delivery of miR-17 lipoplex was performed in collagen induced arthritis model in mice. Clinical, histological and structural effects were studied over the course of arthritis. In depth studies of miR-17 mechanisms of action were performed in primary RA-FLS isolated from RA synovial tissue.

Results: Among others, miR-17-5p expression was reduced in erosive RA miR-17 transfection in arthritic paws significantly reduced clinical inflammation. Moreover, synovial B cells, T cells, macrophages and polynuclear neutrophils infiltrates were significantly reduced. Structural damage was also decreased as shown by a reduction in the number of osteoclasts and erosion score by CT analysis. Pro-inflammatory cytokines of the IL- 6 family, STAT3 target genes and IL-1 $\beta$ expression were also significantly reduced, but not TNF-alpha. miR17 directly targeted the 3'-untranslated region of STAT3 and JAK1. STAT3 and JAK1 mRNA and protein expression were reduced in RA-FLS following miR-17 transfection. STAT3 and JAK1 mRNA and activation of STAT3 as assessed by immunohistochemistry were also reduced in injected paws.

Conclusion: We demonstrate an anti-inflammatory and anti-erosive role of miR-17 in vivo. This effect involves the suppression of the IL-6 family autocrine amplifying loop through the direct targeting of JAK1 and STAT3 as shown in RA-FLS. 